Praxis der Sexualaufklärung und Familienplanung unterstreichen, wo heute folgende Schwerpunkte gesetzt werden $[1,31,32]$ :

- eine über die rein biologische Sexualaufklärung hinausgehende Ausbildung zur Partnerschaft,

- Verbesserung der Kenntnisse zur Anwendung von Verhütungsmethoden,

- Bekanntmachen der neuen Regelung im $\$ 218$ zum Zugang zu Verhütungsmitteln (Kostenübernahme für Kontrazeptiva wie Antibabypille und Spirale für junge Frauen bis zur Vollendung des 20. Lebensjahres).

- Verbesserung der Kenntnisse über Möglichkeiten und Gefahren eines Schwangerschaftsabbruches sowie dessen Finanzierung (nur $18 \%$ sind über die Eigenfinanzierung des $\mathrm{Ab}$ bruchs informiert).

Besondere Zielgruppen sind dabei Jugendliche mit niedrigem Schulabschluß, ausländische Jugendliche und Jugendliche mit gesundheitsschädigendem Verhalten. Nicht nur Schulen, auch an der Jugendarbeit beteiligte Institutionen und Träger, wie Pro familia, Mädchenberatungsstellen, Jugendeinrichtungen und kirchliche Institutionen sollten ihre Arbeit an den o. g. Schwerpunkten orientieren.
Verbesserung der Vorsorge für jugendliche Schwangere und Unterstützung bei der Betreuung und Erziehung des Kindes

- Informationsabende für werdende und junge Mütter/Väter in Schulen, Gemeindezentren und Sozialeinrichtungen anbieten (Elternschulen).

- Vorsorgeuntersuchungen stärker nutzen, um Kenntnisse über Schwangerschaft und Geburt zu vermitteln, verstärkt Einfluß auf das Gesundheitsverhalten bzw. Suchtverhalten der jungen Schwangeren zu nehmen und Unterstützung bei Konflikten (Schule, Familie) anzubieten.

- In der Mütterberatung mehr Kenntnisse zur Entwicklung und Betreuung des Kindes vermitteln (u. a. Vorbeugung von Mißhandlungen). Die Zusammenarbeit mit anderen Institutionen (Jugendhilfe, Sozialeinrichtungen) ist auszubauen.

- Beratungskompetenz des Kinderarztes verbessern.

\section{Aus- und Weiterbildung}

Erzieher, Lehrer und Ärzte sind durch entsprechende Aus- und Weiterbildung in die Lage zu versetzen, auf sexualbiologische und sexualerzieherische Themen besser einzugehen und ein größeres Spektrum von Problemsituationen in bezug auf Partnerschaft in eine Beratung mit einzubeziehen. Eine Untersuchung bei praktischen Ärzten in England zeigte, daß sie auf das Problem einer jugendlichen Schwangerschaft nicht vorbereitet sind [42]. Die Erhöhung der Beratungskompetenz bezieht sich dabei besonders auf Fragen der Familienplanung und Konfliktschwangerschaften. Auch andere Personenkreise (Mitarbeiter der Jugendhilfe, von Jugend- und Sozialeinrichtungen etc). sollten eine entsprechende Weiterbildung erfahren.

\section{Forschung}

Die Einrichtung eines Arbeitsbereiches zur Sexualaufklärung und Familienplanung 1992 in der BZgA hatte eine Schubwirkung im interdisziplinären Diskurs über Sexualität, Kontrazeption und Familienplanung zur Folge. Die im Text erwähnten Ergebnisse zum Sexualverhalten, zum Verhütungsverhalten, zur Aufklärungsarbeit verschiedenster Institutionen sollten Grundlage für neue Präventionstrategien sein und weitere Forschungen anregen.

\section{Literatur:}

Das Litetaturverzeichnis kann bei der Verfasserin Dr. Heidrun Kahl, angefordert werden.

Anschrift der Verfasserinnen:

Dr. Heidrun Kahl, Renate L. Bergmann und Gabricle Röseler, Robert Koch-Institut, Postfach 650280, 13302 Berlin

\title{
Geschlechtsdifferenzen bei Mehrfacherkrankungen
}

\author{
Von J. Grimm, E. Bittner und G. Wiesner
}

\section{Zusammenfassung}

Die gesundheitliche Situation der männlichen Bevölkerung ist trotz insgesamt niedrigerer Prävalenzraten an multimorbiden Zuständen gegenüber der weiblichen Bevölkerung ungünstiger zu beurteilen:

- Im jüngeren Erwachsenenalter haben die Männer höhere Prävalenzraten an multimorbiden Zuständen als die Frauen.

- Die männliche multimorbide Population hat ein um 5,25 Jahre niedrigeres durchschnittliches Krankheitsalter als die weibliche Krankenpopulation.
- Prognostisch ungünstigere multimorbide Profile kommen in der männlichen Krankenpopulation häufiger vor.

$\mathrm{Zu}$ den Folgen der epidemiologischen Transition gehören u. a. ein sozialer Bedeutungswandel von Gesundheitsrisiken, eine Ausdehnung des Krankheitsbegriffs und eine zunehmende Differenzierung pathischer Zustände sowie ein Trend von der Heilung zur Verlängerung des Lebens mit der Krankheit. Eine positiv geleitere Explikation der höheren Morbiditätsraten bei der weiblichen Bevölkerung eröffnet einen inhaltlich konsistenten und logischen Zugang zur Erklärung der gesundheitsbezogenen geschlechtsdifferenten Kenngrößen wie Lebenserwartung und Sterblichkeit.

\section{Einführung}

Frauen und Männer sind hinsichtlich vieler Gesundheitsprobleme unterschiedlich belastet. Die Unterschiede im Gesundheitszustand der männlichen und weiblichen Bevölkerung sind dabei erheblich, wie u. a. an der beträchtlichen Lebenserwartungsdifferenz von 6,5 Jahren zuungunsten der Männer aus der Sterbetafel 1993/95 für die Bundesrepu- 
blik zu ersehen ist. Schon in der intrauterinen Phase besteht eine höhere fötale Sterblichkeit des männlichen $\mathrm{Ge}$ schlechts. In jedem Lebensalter setzt sich die höhere Sterblichkeit des männlichen Geschlechts fort.

Andernteils klagen Frauen häufiger über schlechtes Befinden. Sie führen mehr physische und psychische $\mathrm{Be}$ schwerden an. Eine höhere Krankheitsintensität der weiblichen Bevölkerung gegenüber der männlichen Bevölkerung läßt sich nicht nur für alle Altersgruppen und für die deutsche Bevölkerung in Ost und in West nachweisen, sondern auch für alle sozialen Schichten [1]. Frauen sind auch das "gesundheitsbewußtere Geschlecht «. Beispielsweise ist die Ernährung von Frauen abwechslungs- und vitaminreicher [2-4], dementsprechend ist ein deutlich höherer Anteil von Männern jüngeren Alters adipös [5]. Frauen gehen auch häufiger zum Arzt $[6,7]$, nutzen gegenüber Männern vermehrt die von den Krankenkassen angebotenen Früherkennungsuntersuchungen [8] und nehmen mehr Medikamente ein.

Die Gründe für diese gesundheitliche Ungleichheit sind vielschichtig; sie sind schon mehrfach erörtert und beschrieben worden, aber bisher weitestgehend ungeklärt geblieben. Für die Herausbildung von gesundheitsbezogenen Ungleichstrukturen zwischen den Geschlechtern sind ohne Zweifel soziale, kulturelle, genetische und biologische Faktoren maßgebend. Die geschlechtsdifferenten Gefährdungsstrukturen, Ungleichheiten und Verhaltensweisen im Umgang mit Krankheiten lassen nicht nur Unterschiede in der Häufigkeit und Höhe des Krankseins erwarten (quantitative Aspekte), sondern auch in der Art des Krankseins selbst (qualitative Aspekte). Neben den direkt geschlechtsgebundenen Beschwerde- und Erkrankungsformen (z. B. das Prämenstruelle Syndrom oder das Zervixkarzinom bei der Frau; eine Orchitis/Epididymitis oder das Prostatakarzinom beim Mann) sind bei einer Vielzahl von Krankheiten geschlechtsdifferente Inzidenz- und Prävalenzraten zu verzeichnen. Beispielsweise erleidet die männliche Population im Alter von 25 bis unter 75 Jahren etwa dreimal häufiger einen Herzinfarkt als die gleichaltrige weibliche Population [9]. Bei Frauen ist wiederum ein höherer Anteil von depressiven und neurotischen $\mathrm{Er}$ krankungen oder Osteoporose zu beobachten $[10,11]$. Die geschlechtsspezifi- sche Daseinsform des Krankseins und das damit verbundene Umfeld, die schwierigen Abgrenzungen von Gesundheit und Krankheit in Abhängigkeit von verschiedenen Lebensphasen und unterschiedlichen Sozialisationen der Geschlechter, die geschlechtsdifferenten Wege zur Diagnose sowie die sich daraus ableitenden Konsequenzen (bis hin zu diagnostisch-therapeutischen Entscheidungen), die geschlechtsgebundene Konzeptualisierung von $\mathrm{Ge}$ sundheit und Krankheit etc. sind unzureichend geklärt. Das »anders Kranksein « müßte sich je nach der Geschlechtszugehörigkeit auch bei den Mehrfacherkrankungen widerspiegeln. Dazu liegen bisher kaum Erkenntnisse vor.

\section{Material und Methodik}

Die Untersuchungsergebnisse basieren auf den Daten des Gesundheitssurveys Ost, der 1991/92 in den neuen Bundesländern durchgeführt wurde. Die repräsentative Stichprobe des Surveys Ost setzte sich aus 2617 Personen zwischen 18 und 79 Jahren zusammen, verteilt auf 50 Sample Points. Die Teilnehmerrate am Survey lag bei $70 \%$. Die zufällige Auswahl der Sample Points erfolgte entsprechend der Verteilung der Gemeindegrößenklassen. Die Namen der zur Stichprobe gehörigen Einwohner der Ortschaften wurden zufällig aus der Einwohnerkartei ausgewählt (geschichtete Zufallsauswahl). Die so gewonnene Stichprobe gestattet zuverlässige Rückschlüsse auf die gesundheitliche Situation der ostdeutschen Bevölkerung. In der Untersuchung wurden $u$. a. epidemiologisch relevante Zustände des Krankseins als jährliche Prävalenzrate (annual prevalence rate) erfaßt. Analog können auch relevante Zustände des Nicht-Krankseins als Prävalenzen für den Zeitraum eines Jahres ausgewiesen werden.

Auf die Einbeziehung von Lebenszeit-Prävalenzen wurde aus folgenden Gründen verzichtet:

- Weniger ernsthafte Krankheitsepisoden, die beispielsweise bei älteren Probanden in der Jugend bzw. jüngerem Erwachsenenalter aufgetreten sein können, werden in der Befragungssituation bevorzugt vergessen;

- zeitlich weit zurückliegende Beschwerden/Symptome brauchen keine Relevanz für die aktuelle gesundheitliche Situation der Probanden zu haben.
Die jährlichen Prävalenzraten wurden in den Gesundheitssurveys bisher nur in Ostdeutschland erfaßt. Daher konnte nur der Gesundheitssurvey Ost für die Datenanalyse genutzt werden. Aus den Surveydaten wurden die Prävalenzen von 36 verschiedenen Krankheiten, Beschwerden und Symptomen einer Auswertung zugeführt.

Dabei handelt es sich um folgende epidemiologisch relevante Zustände:

\section{Kenn-Nr.}

1 Durchblutungsstörungen am Herzen, Angina pectoris

2 Herzinfarkt

3 Herzschwäche (Herzinsuffizienz, "Herzasthma«)

4 Durchblutungsstörungen im Gehirn

5 Zuckerkrankheit, Diabetes

6 Schlaganfall

7 Varikose ("Krampfadern«), Thrombose, Phlebitis (inkl. Thrombophlebitis)

8 Durchblutungsstörungen in den Beinen (außer Krampfadern)

9 Bluthochdruck, Hypertonie

10 zu niedriger Blutdruck, Hypotonie

11 starkes Übergewicht, Fettsucht

12 Gicht, Harnsäureerhöhung

13 Hypercholesterinämie, erhöhtes Cholesterin, Hyperlipidämie, erhöhte Blutfette

14 Gelenkrheumatismus, chron. Gelenkentzündung, Arthritis, Arthrose

15 Lumbago (»Hexenschuß«), Ischiassyndrom (Ischias)

16 Osteoporose (Knochenschwund)

17 Bandscheibenschaden (inkl. Bandscheibenprolaps, Chondrosis intervertebralis, Osteochondrosis intervertebralis, Zustand nach Wirbelsäulenfrakturen)

18 Leberentzündung, akute und chronische Hepatitis, Fettleber

19 Leberverhärtung, Leberzirrhose

20 Cholelithiasis/Cholezystitis (Gallensteine, Gallenblasenentzündung)

21 Magen-, Zwölffingerdarmgeschwür, Ulcus

22 Gastritis (Magenschleimhautentzündung)

23 Struma (Kropf), andere Schilddrüsenkrankheiten

24 Entzündung oder Steine der Blase, der Niere, der Harnwege

25 Verdauungsbeschwerden 
26 Heuschnupfen

27 andere Allergien

28 Körperbehinderungen der oberen Gliedmaßen oder Schulter z. B. Lähmungen, Fehlen von Gliedmaßen oder Teiten, Fehibildungen, Gelenkversteifungen)

29 Körperbehinderungen der unteren Gliedmaßen oder Hüfte (z. B. Lähmungen, Fehlen von Gliedmaßen oder Teilen, Fehlbildungen, Gelenkverstellungen)

30 Körperbehinderungen $d$. Wirbelsäule (z. B. Verkrümmungen, Versteifungen oder Fehlbildungen, nicht Muskelverspannungen)

31 Schenkelhalsfraktur

32 Glaukom (grüner Star)

33 Hormonstörungen

34 psychische Störungen oder Krankheiten

35 Hauterkrankungen

36 Krebskrankheiten

Die Personen der Stichprobe mit Mehrfacherkrankungen im Zeitraum eines Jahres wurden als Multimorbidität definiert. Hierbei bestand das Problem wie man die hohe Anzahl der möglichen Krankheitsgruppen $\left(2^{36}-37\right)$, die man aus 36 verschiedenen Krankheiten, Beschwerden und Symptomen bilden kann, auf ein vernünftiges $M a ß$ an zu untersuchenden diagnostischen Gruppierungen/Krankheitskombinationen zu reduzieren vermag. Eine Möglichkeit der Reduktion bestand in der Bildung von Basiskrankheitsgruppen. Unter einer Basiskrankheitsgruppe wurde eine bestimmte Anzahl von definierten Krankheiten verstanden, die bei einem größeren Anteil der Bevölkerung prävalent waren. Bei diesem Bevölkerungsanteil können im betrachteten Zeitraum eines Jahres auch noch die anderen Krankheiten aus dem Reservoir der vorgegebenen 36 Krankheiten auftreten. Wenn diese Krankheiren niedrige Bestands häufigkeiten hatten, wurden sie in den Basiskrankheitsgruppen nicht berücksichtigt. Bei der Aufstellung der Basiskrankheitsgruppen wurden jeweils zwei, drei, vier, funf oder sechs verschiedene Krankheiten, Beschwerden und Symptome erfaßt. Nach der Anzahl der Basiskrankheitsgruppen, konstituierenden Krankheiten, Beschwerden und Symptome wurden sie als zweier, dreier, vierer, fünfer oder sechser $\mathrm{Ba}$ sisgruppen bezeichnet. Die einzelnen Krankheiten, Beschwerden und Symptome wurden im weiteren fortan unter dem Begriff »Krankheit « geführt.
Un diese Vorhergehensweise durchführen zu können, wurde zunächst ein nicht-hierarchisches Clusterverfahren nach der $\mathrm{k}$-means Methode von MacQueen [12] angewandt. Dieses Verfahren ist durch die "Fastclus Procedure" von SAS realisiert und wurde auch in Hartigan [13] beschrieben. Der Algorithrnus wurde auf die durch Indikatorvariablen verschlüsselten Krankheiten angewandt (alle Indikatorvariablen wurden als ein Zahlenschema, ein Vektor, betrachtet).

Der Algorithmus besteht aus folgenden, wiederholt durchzuführenden Schritten:

1. Man erzeugt eine Anfangspartition mit k Clustern.

2. Beginnend mit dem 1. Objekt im 1. Cluster wurden für alle Objekte die euklidischen Distanzen zu allen $\mathrm{Clu-}$ sterschwerpunkten bestimmt.

3. Trifft man auf ein Objekt, das zu dem Schwerpunkt des eigenen Clusters eine größere Distanz aufweist als zum Schwerpunkt eines anderen Clusters, wird dieses Objekt in dieses Cluster verschoben.

4. Die Schwerpunkte der beiden durch diese Verschiebung veränderten Cluster werden neu berechnet.

5. Man wiederholt Schritt 2 bis Schritt 4 bis sich jedes Objekt in einem Cluster befindet, zu dessen Schwcrpunkt es im Vergleich zu den übrigen Clustern die geringste Distanz aufweist.

Da der euklidische Abstand von Vektoren aus Indikatorvariablen die entscheidende Größe für das Finden der Cluster ist, mußte aufgrund des Verfahrens zu erwarten sein, daß die Mitglieder eines Clusters so bestimmt werden, daß bei möglichst vielen von ihnen gleiche mit " « gekennzeichneze sowie gleiche mit »O gekennzeichnete Krankheiten der 36 Krankheiten auftreten. Dabei bedeutet die Zuordnung einer »I « für eine Krankheit bei einem Clustermitglied, daß die Krankheit im betrachteten Zeitraum bei ithm vorhanden war. Die $\mathrm{Zu}$ ordnung einer $\cong$ « bedeutet das Gegenteil.

Die sechs Krankheiten, deren einzelne Anteile bei den Mitgliedern eines Clusters am höchsten waren, dienten zur Konstruktion der Basisgruppen. Die zwei Krankheiten mit den höchsten Einzelanteilen bei den jeweiligen Clustermitgliedern wurden als 2er Basisgruppe, die drei Krankheiten mit den höchsten Einzelanteilen bei den jeweiligen Clustermitgliedern als 3er Basis- gruppe usw. definiert. Auf diese Weise wurden aus jedem Cluster fünf mögliche Krankheitsbasisgruppen abgeleitet. Als besonders relevant wurden dann diejenigen der so bestimmten Basisgruppen angesehen, die hinsichtlich der Anteile an der Population, der jährlichen Arztbesuche und der täglichen Medikamenteneinnahme über dem jeweiligen Durchschnitt der Gruppe gleicher Ordnung lagen (dabei wurde unter dem Begriff Ordnung einer Krankheitsbasisgruppe die Anzahl der die Basisgruppen definierenden Krankheiten verstanden). Bezüglich der gesamten drei Parameter wurde dann eine Rangordnung der 17 Basisgruppen ermittelt (eine detailliertere Beschreibung der Vorgehensweise ist dem ausführlichen Bericht zum Forschungsprojekt 19, 1997 [14] zu entnehmen).

Innerhalb einer Basiskrankheitsgruppe befindet sich die Menge an Personen, die von einer bestimmten Anzahl definierter Zustände des Krankseins (zwei bis sechs Krankheiten) betroffen sind. Die gleiche Person kann in einer oder mehreren der 17 Basiskrankheitsgruppen enthalten sein.

Zur Erfassung der multimorbiden Krankenpopulation der 17 Basiskrankheitsgruppen wurde nochmals eine personenbezogene Auswertung vorgenommen.

Gleichzeitig wurden für die ostdeutsche Gesamtbevölkerung auf der Basis der 36 Krankheiten (unabhängig von der Auswahl der 17 Basiskrankheitsgruppen) die jährlichen Prävalenzraten nach der Anzahl der Krankheiten berechnet.

\section{Ergebnisse}

Innerhalb einer Zwölf-Monatsperiode waren $39,3 \%$ der ostdeutschen männlichen Bevölkerung und 51,2 Prozent der weiblichen Bevölkerung von mindestens zwei Krankheiten betroffen (bezogen auf die Anzahl der 36 vorgegebenen Krankheiten und das Alter von 18 bis unter 80 Jahren). Die epidemiologischen Intervalle des mehrfachen Krankseins varïeren beträchtlich nach der Anzahl der Krankheiten und der Geschlechtszugehörigkeit (Tab. 1, Abb. 1).

Die weibliche Population hat durchgehend höhere Bestandshäufigkeiten des Krankseins aufzuweisen als die männliche Population. Mit steigender Anzahl der Krankheiten bzw. Zunahme der Multimorbidität werden die Differenzen in den Prävalenzraten zwischen Männern und Frauen immer größer. 

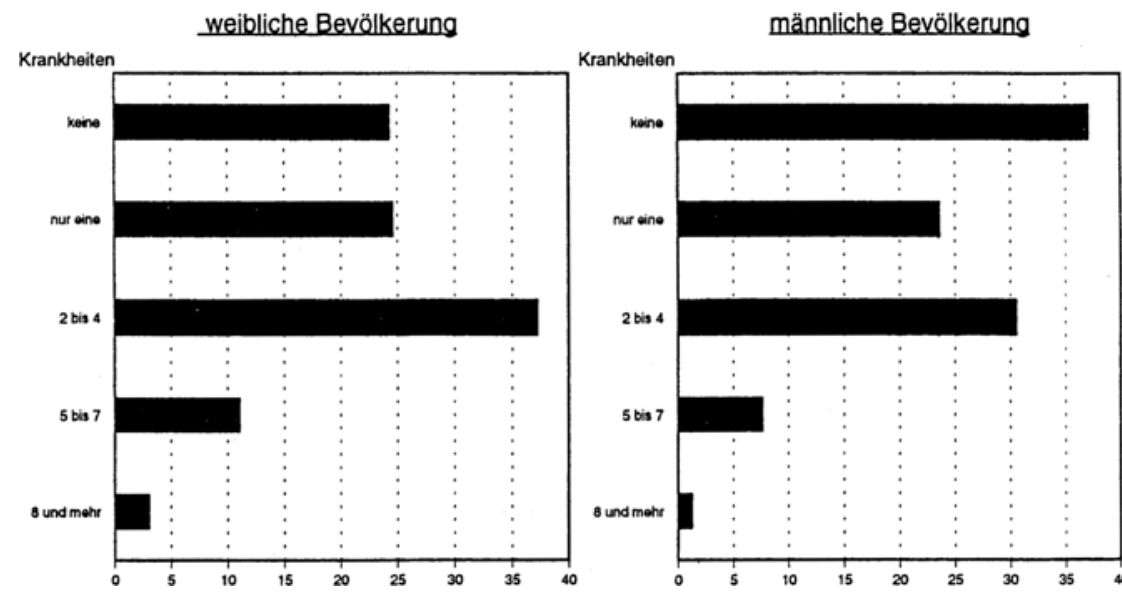

weiblichen Bevölkerung. Danach haben die Frauen mit steigendem Lebensalter zunehmend höhere Prävalenzraten. Im Alter von 70 bis unter 80 Jahren besteht eine Relation betroffener Frauen zu Männern von 1,7:1. Im Unterschied zu der weiblichen Bevölkerung weisen die 70-bis 79jährigen betagten Männer wieder eine niedrigere Prävalenzrate gegenüber der vorhergehenden jüngeren Altersgruppe der 60- bis 69jährigen auf. Das durchschnittliche Krankheitsalter der männlichen multimorbiden Population beträgt 57,43 Jahre, während in der repräsentativen Bevölkerungsstichprobe des Surveys das durchschnittliche Lebensalter der männlichen Bevölkerung bei 44,23 liegt; bei der weiblichen Population sind die entsprechenden Werte 62,68 für das durchschnittliche

Abbildung 1: Anzahl unterschiedlicher Krankheiten innerhalb eines Jahres (in Prozent); 18- bis 80jährige Bevölkerung in den neuen Bundesländern, 1991/92.

Während beim Auftreten von »nur einer Krankheit « im Jahresverlauf unerhebliche Unterschiede in den geschlechtsdifferenten Prävalenzraten bestehen, beträgt das Verhältnis von vielfachkranken Frauen zu Männern bei acht und mehr Krankheiten fast 2,5:1.

Bei den jährlichen Prävalenzraten des Nicht-Krankseins (bezogen auf das Vorkommen der 36 ausgewählten Krankheiten) sind die Verhältnisse genau umgekehrt: mehr als jeder dritte Mann $(37,1 \%)$, aber weniger als jede vierte Frau (24,2\%) weist keine der vorgegebenden Diagnosen, Beschwerden und Symptome innerhalb einer ZwölfMonatsperiode auf.

Bei beiden Geschlechtern nimmt die Höhe der jährlichen Prävalenzraten mir der Zahl der Krankheiten kontinuierlich ab: Die Menge prävalenter Fälle mit nur einer oder zwei Krankheiten in der Bevölkerung ist erheblich höher als die Bestandsmenge an Vielfachkranken mit drei oder mehr Krankheiten (Tab. 1). Immerhin hat jede fünfte Frau (20,2 \% der weiblichen Bevölkerung) und fast jeder siebte Mann (13,7 \% der männlichen Bevölkerung) vier verschiedene Krankheiten und mehr im Jahr.

In der Tabelle 2 sind die altersspezifischen Prävalenzraten der multimorbiden Population aufgeführt, die sich aufgrund der modifizierten Clusteranalyse nur auf die Auswahl von 17 Basiskrankheitsgruppen beschränkt.

Im jüngeren Erwachsenenalter sind bis zum 40. Lebensjahr die männlichen Prävalenzraten an multimorbiden $\mathrm{Zu}$ ständen höher als die der gleichaltrigen
Tabelle 1: Jährliche Prävalenzraten (annual prevalence rate) je 1000 der Bevölkerung gleichen Geschlechts nach der Anzahl der Krankheiten"; 18- bis 80jährige deutsche Bevölkerung in den neuen Bundesländern 1991/92

\begin{tabular}{lccc}
\hline & \multicolumn{2}{c}{ Jährliche Prävalenzrate je 1000 der Bevölkerung } \\
$\begin{array}{l}\text { Anzahl der } \\
\text { Krankheiten }\end{array}$ & männlich & weiblich & $\begin{array}{l}\text { Quotient } \\
\text { w/m }\end{array}$ \\
\hline 1 & 235,5 & 245,3 & 1,04 \\
2 & 160,3 & 193,7 & 1,21 \\
3 & 95,7 & 116,2 & 1,21 \\
4 & 49,1 & 62,4 & 1,27 \\
5 & 45,8 & 56,7 & 1,24 \\
6 & 18,8 & 34,4 & 1,64 \\
7 & 11,4 & 18,7 & 1,64 \\
8 und mehr & 12,3 & 30,1 & 2,45
\end{tabular}

Krankheiten

Die Unterschiede zwischen den geschlechtsspezifischen Prävalenzraten sind nach Chi ${ }^{2}$-Test signifikant $(\mathrm{P}=0,001)$.

* Zahl der cinzelnen nosologischen Entitäten (nicht die Zahl einzelner Krankheitsepisoden der gleichen Krankheit), die im Verlauf einer vollen Jahresperiode aufgetreten sind.

Tabelle 2: Altersspezifische jährliche Prävalenzraten (age-specific annual prevalence rate) der 18- bis 80jährigen multimorbiden Population (basierend auf den 17 Basiskrankheitsgruppen)*; neue Bundesländer 1991/92

\begin{tabular}{|c|c|c|c|}
\hline \multicolumn{4}{|c|}{ Jährliche Prävalenzrate je 1000 der Bevölkerung } \\
\hline $\begin{array}{r}\text { Alter } \\
\text { (Jahre) }\end{array}$ & männlich & weiblich & $\begin{array}{r}\text { Quotient } \\
w / m\end{array}$ \\
\hline $18-29$ & 8,77 & 3,66 & 0,42 \\
\hline $30-39$ & 27,78 & 9,32 & 0,34 \\
\hline $40-49$ & 49,55 & 51,50 & 1,04 \\
\hline $50-59$ & 130,91 & 132,29 & 1,01 \\
\hline $60-69$ & 253,25 & 281,41 & 1,11 \\
\hline $70-79$ & 214,29 & 363,64 & 1,70 \\
\hline$\sum 18-79$ & 88,31 & 104,73 & 1,19 \\
\hline
\end{tabular}

* Personenbezogene Bestandsmenge an multimorbiden Zuständen; jede Person wird nur einmal gezählt, unabhängig ob sie an einer oder mehreren der 17 Basiskrankheitsgruppen beteiligt ist. 
Übersicht 1: Struktur der Mehrfacherkrankungen nach Diagnosegruppierungen (multimorbides Profil)

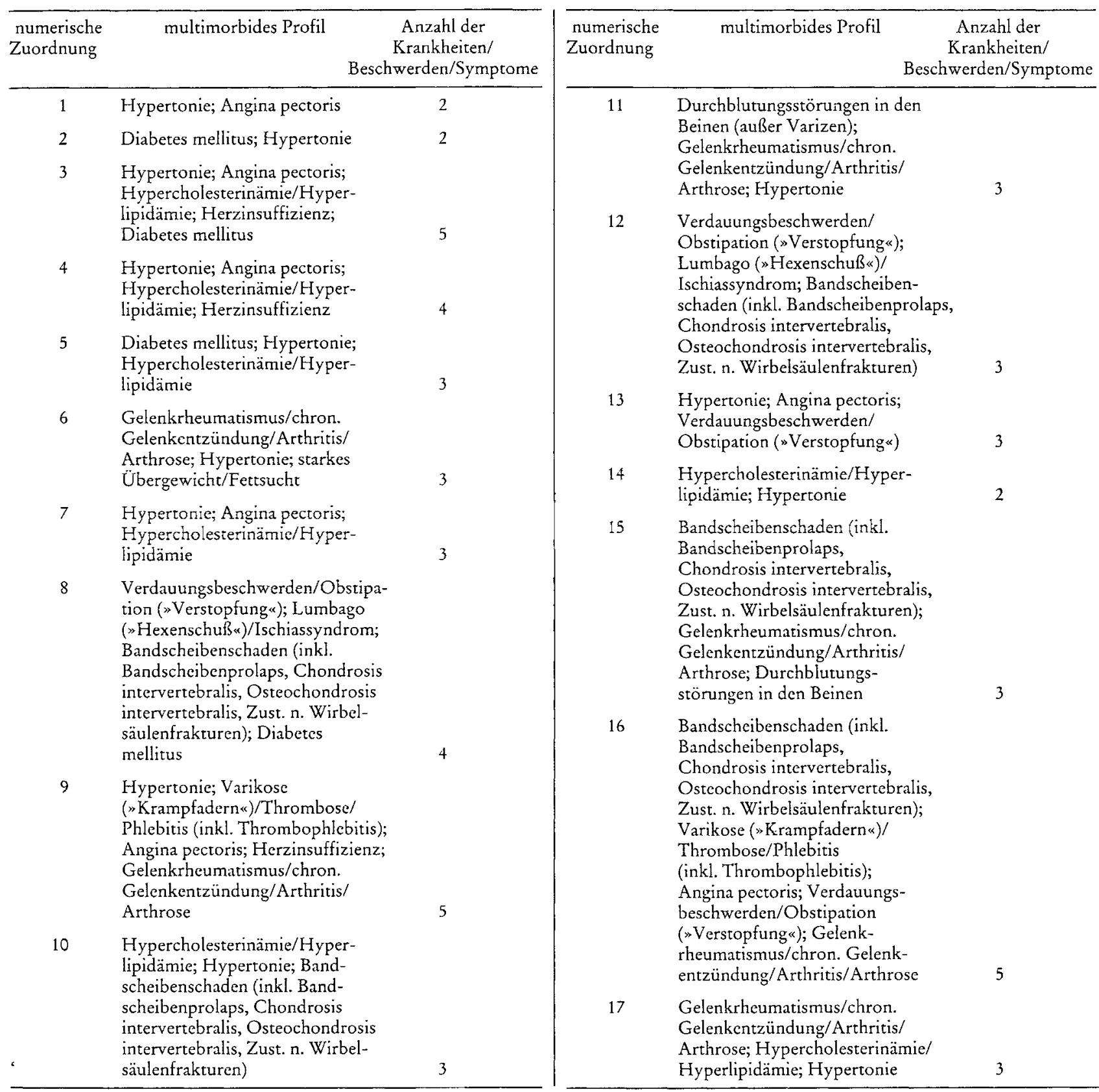

Krankheitsalter und 45,10 für das durchschnittliche Lebensalter. Demnach liegt für die multimorbide Population das durchschnittliche Krankheitsalter der männlichen Krankenpopulation um 13,20 Lebensjahre und das der weiblichen Krankenpopulation um 17,56 Lebensjahre höher als das jeweilige durchschnittliche Lebensalter der 18- bis 80jährigen Bevölkerung. Die männliche multimorbide Population hat ein um 5,25 Lebensjahre niedrigeres durchschnittliches Krankheitsalter als die ent- sprechende weibliche Krankenpopulation.

In der Übersicht 1 ist die Struktur der Mehrfacherkrankungen nach den ausgewählten 17 Diagnosengruppierungen (multimorbides Profil) aufgeführt. Von den 36 unterschiedlichen nosologischen Entitäten sind nur zwölf Krankheiten/Beschwerden/Symptome an der Konstituierung dieser relevanten Basiskrankheitsgruppen beteiligt.
Von den theoretisch mehr als 68,7 Milliarden denkbaren Krankheitskombinationen aus 36 unterschiedlichen Krankheiten repräsentieren diese 17 Basiskrankheitsgruppen mehr als ein Fünftel der Gesamtmorbidität von Zuständen des Krankseins mit zwei und mehr Krankheiten (siehe Tab. 1).

Die Bluthochdruckkrankheit trägt mit Abstand am häufigsten an der Formierung von Mehrfacherkrankungen inner- 
halb der Bevölkerung bei. Auch die koronare Herzkrankheit »Angina pectoris“, die Fettstoffwechselstörungen »Hypercholesterinämie u und »Hyperlipidämie", Arthropathien (Gelenkrheumatismus, chronische Gelenkentzündung, Arthritis, Arthrose) und Dorsopathien (Bandscheibenschaden, Bandscheibenprolaps, Chondrosis intervertebralis etc.) sind häufig Bestandteile von Krankheitskombinationen, die bevölkerungsbezogen relevanten Mengen des Krankseins zuzuordnen sind. Dagegen findet man derartig schwerwiegende und/oder prognostisch ungünstig zu bewertende Krankheiten/Zustände, wie beispielsweise Myokardinfarkt, onkologische Leiden, Apoplektischer Insult, Leberzirrhose, Schenkelhalsfraktur nicht in den bevölkerungsrelevanten Krankheitskombinationen vor. Überraschenderweise treten auch in der Bevölkerung weit verbreitete gesundheitliche Problemstellungen, wie Allergien, Hauterkrankungen, psychische Störungen oder psychiatrische Krankheiten, Hypotonien oder Osteoporose nicht in der multimorbiden Ursachenstruktur der 17 Basiskrankheitsgruppen auf.

Die modifizierte Clusteranalyse hat am häufigsten Krankheitskombinationen erstellt, die sich aus drei verschiedenen Krankheiten zusammensetzen (neun von 17 Basiskrankheitsgruppen). Die jährlichen Prävalenzraten an Mehrfacherkrankungen nehmen mit der Anzahl der Krankheiten (Typ der Basisgruppe) innerhalb der einzelnen Diagnosegruppierungen $\mathrm{ab}$; die zweier $\mathrm{Ba}$ sisgruppen haben die höchsten, die fün-

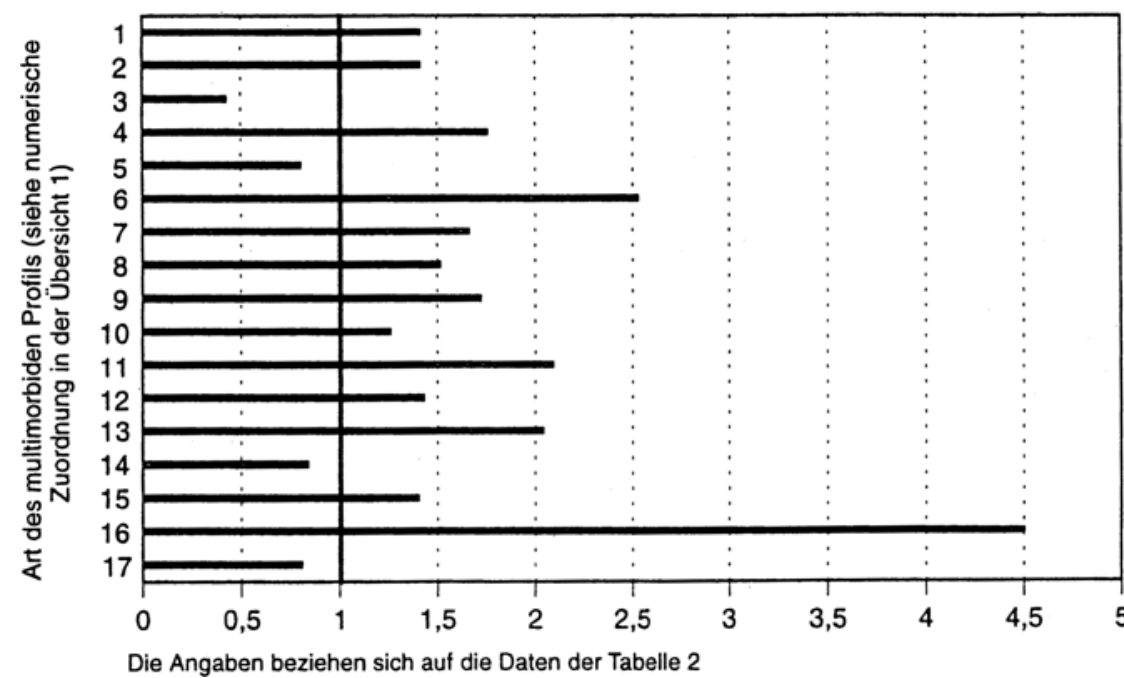

Abbildung 2: Verhältnis mehrfacherkrankter Frauen zu Männern (=1) je 1000 der Bevölkerung gleichen Geschlechts. $1991 / 92$ nicht anwendbar. fer Basisgruppen die niedrigsten Prävalenzraten. Diabetes mellitus, Hochdruckkrankheit, Angina pectoris und Fettstoff wechselstörungen sind an keinen bestimmten Typ der Basisgruppen gebunden. Die Krankheitsbilder Herzinsuffizienz und Varikose/Thrombose/ Phlebitis kommen bevorzugt in Dia-
Tabelle 3: Multimorbide Ursachenstruktur - jährliche Prävalenzraten (annual prevalence rate) je 1000 der Bevölkerung gleichen Geschlechts nach einzelnen Diagnosengruppierungen; 18- bis 80jährige deutsche Bevölkerung in den neuen Bundesländern,

\begin{tabular}{ccccc}
\hline $\begin{array}{c}\text { Multimorbides } \\
\text { Profil* }\end{array}$ & $\begin{array}{c}\text { jährliche Prävalenzraten** je } 1000 \text { der Bevölkerung } \\
\text { männlich }\end{array}$ & weiblich & $\begin{array}{c}\text { P-Werte für Chi } \\
\text { Signifikanztest }\end{array}$ & $\begin{array}{c}\text { Prävalenzdifferenz } \\
\text { w m }\end{array}$ \\
\hline 1 & 36,0 & 50,9 & 0,063 & $+14,9$ \\
2 & 21,3 & 30,1 & 0,155 & $+8,8$ \\
3 & 3,3 & 1,4 & $0,327 \times$ & $-1,9$ \\
4 & 4,1 & 7,2 & 0,297 & $+3,1$ \\
5 & 9,0 & 7,2 & 0,602 & $-1,8$ \\
6 & 7,4 & 18,7 & 0,012 & $+11,3$ \\
7 & 6,5 & 10,8 & 0,249 & $+4,3$ \\
8 & 3,3 & 5,0 & 0,490 & $+1,7$ \\
9 & 2,5 & 4,3 & $0,42 \times$ & $+1,8$ \\
10 & 7,4 & 9,3 & 0,582 & $+1,9$ \\
11 & 6,5 & 13,6 & 0,073 & $+7,1$ \\
12 & 6,5 & 9,3 & 0,426 & $+2,8$ \\
13 & 4,9 & 10,0 & 0,132 & $+5,1$ \\
14 & 36,8 & 30,8 & 0,400 & $-6,0$ \\
15 & 8,2 & 11,5 & 0,39 & $+3,3$ \\
16 & 0,8 & 3,6 & $0,139 \times$ & $+2,8$ \\
17 & 9,8 & 7,9 & 0,599 & $-1,9$ \\
\hline
\end{tabular}

* Siehe numerische Zuordnung in der Übersicht 1; ** Der Bezug zur Person ist nur in der jeweiligen Diagnosengruppierung gegeben. $x$ Chi²-Test aufgrund zahlenmäßiger Besetzung

gnosegruppierungen vor, die eine relativ hohe Anzahl von Krankheiten beinhalten (hier die vierer und fünfer Basisgruppen).

Die weibliche Bevölkerung hat überwiegend deutlich höhere Prävalenzraten bei den einzelnen Diagnosegruppierungen als die männliche Bevölkerung (Abb. 2).

Bemerkenswert ist, daß insbesondere Krankheitskombinationen mit Dorsopathien, Arthropathien, Venenleiden und Verdauungsbeschwerden/Obstipationen bei den Frauen auftreten. Große Unterschiede zwischen Frauen und Männern sind insbesonders bei zwei multimorbiden Strukturen zu verzeichnen: In der fünfer Basisgruppe 16 (Dorsopathien, Arthropathien, Venenerkrankungen, Angina pectoris, Verdauungsbeschwerden) beträgt das Verhältnis betroffener Frauen zu Männern 4,5:1; in der dreier Basisgruppe 6 (Arthropathien, starkes Übergewicht, $\mathrm{Hy}$ pertonie) entsprechend 2,5:1 (Tab. 3).

Lediglich bei vier Diagnosegruppierungen der 17 Basiskrankheitsgruppen hat die männliche Bevölkerung höhere Prävalenzraten. Der größte Unterschied besteht hier bei der fünfer Basisgruppe 3 (Angina pectoris, Diabetes mellitus, Herzinsuffizienz, Hypertonie, Hyper- 
Tabelle 4 a: Multimorbide Ursachenstruktur: durchschnittliches Krankheitsalter der männlichen Krankenpopulationen (Lebensalter in vollendeten Jahren), Standardabweichung und Altersspanne (minimales Krankheitsalter = Minimum; maximales Krankheitsalter $=$ Maximum) der einzelnen multimorbiden Populationen; neue Bundesländer $1991 / 92$

\begin{tabular}{|c|c|c|c|c|}
\hline \multicolumn{5}{|c|}{ männliche Krankenpopulationen } \\
\hline $\begin{array}{l}\text { Multimorbides } \\
\text { Profil* }\end{array}$ & $\begin{array}{l}\text { Krankheits- } \\
\text { alter } \varnothing\end{array}$ & $\begin{array}{l}\text { Standard- } \\
\text { abweichung }\end{array}$ & Minimum & Maximum \\
\hline 1 & 61,73 & 9,37 & 30,00 & 77,00 \\
\hline 2 & 62,38 & 6,59 & 50,00 & 78,00 \\
\hline 3 & 62,25 & 2,06 & 60,00 & 65,00 \\
\hline 4 & 60,20 & 4,92 & 52,00 & 65,00 \\
\hline 5 & 60,90 & 5,24 & 52,00 & 69,00 \\
\hline 6 & 57,44 & 8,35 & 51,00 & 72,00 \\
\hline 7 & 59,75 & 5,15 & 52,00 & 66,00 \\
\hline 8 & 66,50 & 7,05 & 62,00 & 77,00 \\
\hline 9 & 72,33 & 6,43 & 65,00 & 77,00 \\
\hline 10 & 54,33 & 8,23 & 39,00 & 64,00 \\
\hline 11 & 56,63 & 13,33 & 34,00 & 77,00 \\
\hline 12 & 62,50 & 9,62 & 47,00 & 77,00 \\
\hline 13 & 69,33 & 9,67 & 53,00 & 77,00 \\
\hline 14 & 52,84 & 10,76 & 27,00 & 71,00 \\
\hline 15 & 57,50 & 16,27 & 23,00 & 78,00 \\
\hline 16 & 77,00 & $\bullet$ & 77,00 & 77,00 \\
\hline 17 & 53,33 & 8,57 & 34,00 & 66,00 \\
\hline
\end{tabular}

* Siehe numerische Zuordnung in der Übersicht 1.

\section{Diskussion}

Die Ungleichheit in der Prävalenz multimorbider Zustände ist zwischen den Geschlechtern erheblich: Die weibliche Bevölkerung hat insgesamt höhere Bestandshäufigkeiten an Mehrfachkrankheiten als die männliche Bevölkerung. Der gesundheitsbezogene Handlungsbedarf scheint demnach mehr für die weibliche Bevölkerung gegeben zu sein, um diese "gesundheitliche Benachteiligung" wenigstens ausgleichen zu können.

Eine nähere Betrachtung der Struktur der multimorbiden Population läßt aber folgende Merkmale erkennen:

- Im jüngeren Erwachsenenalter haben die Männer höhere Prävalenzraten an multimorbiden Zuständen als die Frauen.

- Der Trend steigender Prävalenzraten an multimorbiden Zuständen mit zunehmendem Lebensalter wird in der Altersgruppe der 70- bis unter 80jährigen Männer unterbrochen - in diesem betagten Alter nehmen die Prävalenzen bei den Frauen weiterhin $\mathrm{zu}$, bei den Männern hingegen $a b$.

- Die männliche multimorbide Population hat ein um 5,25 Jahre niedrigeres durchschnittliches Krankheitsalter als die weibliche Krankenpopulation. cholesterinämie), wo Männer eine 2,4 höhere Prävalenzrate als Frauen aufweisen.

In der Tabelle $4 \mathrm{a}$ und $4 \mathrm{~b}$ sind die durchschnittlichen Werte des Krankheitsalters für die Basiskrankheitsgruppen aufgeführt. Danach differiert das durchschnittliche Krankheitsalter zwischen Männern und Frauen je nach der Art der Mehrfacherkrankung im Intervall von 0,06 und 10,00 Jahren. Die Spannweite zwischen dem höchsten und niedrigsten durchschnittlichen Krankheitsalter beträgt bei den Männern 24,16 Jahre und bei den Frauen 12,12 Jahre. In elf der 17 Basisgruppen haben die Männer ein niedrigeres durchschnittliches Krankheitsalter als die Frauen. Ein höheres durchschnittliches Krankheitsalter korreliert bei beiden Geschlechtern positiv mit den Herzkrankheiten Angina pectoris und Herzinsuffizienz. Hypertonie und Hypercholesterinämie/Hyperlipidämie sind Krankheiten, die in den Basiskrankheitsgruppen der Männer schon in einem relativ niedrigeren durchschnittlichen Krankheitsalter anzutreffen sind; bei den Frauen sind es bevorzugt die Arthropathien.
Tabelle 4 b: Multimorbide Ursachenstruktur: durchschnittliches Krankheitsalter der weiblichen Krankenpopulationen (Lebensalter in vollendeten Jahren), Standardabweichung und Altersspanne (minimales Krankheitsalter = Minimum; maximales Krankheitsalter = Maximum) der einzelnen multimorbiden Populationen; neue Bundesländer 1991/92 weibliche Krankenpopulationen

\begin{tabular}{ccccc}
$\begin{array}{c}\text { Multimorbides } \\
\text { Profil* }\end{array}$ & $\begin{array}{c}\text { Krankheits- } \\
\text { alter } \varnothing\end{array}$ & $\begin{array}{c}\text { Standard- } \\
\text { abweichung }\end{array}$ & Minimum & Maximum \\
\hline 1 & 65,34 & 8,19 & 42,00 & 79,00 \\
2 & 64,52 & 9,55 & 41,00 & 79,00 \\
3 & 69,50 & 12,06 & 61,00 & 78,00 \\
4 & 67,50 & 5,42 & 61,00 & 78,00 \\
5 & 63,90 & 7,22 & 56,00 & 78,00 \\
6 & 57,38 & 13,71 & 22,00 & 77,00 \\
7 & 66,53 & 6,47 & 56,00 & 79,00 \\
8 & 61,29 & 8,36 & 52,00 & 71,00 \\
9 & 67,50 & 10,54 & 49,00 & 79,00 \\
10 & 61,85 & 10,49 & 51,00 & 79,00 \\
11 & 63,95 & 9,95 & 47,00 & 79,00 \\
12 & 61,08 & 12,17 & 36,00 & 80,00 \\
13 & 69,14 & 4,52 & 63,00 & 77,00 \\
14 & 60,93 & 8,56 & 48,00 & 79,00 \\
15 & 66,81 & 9,83 & 49,00 & 79,00 \\
16 & 67,00 & 7,11 & 56,00 & 75,00 \\
17 & 62,64 & 8,89 & 52,00 & 78,00 \\
\hline
\end{tabular}

* Siehe numerische Zuordnung in der Übersicht 1. 
- Während die Männer bei den meisten diagnostischen Gruppierungen an Mehrfacherkrankungen zum Teil deutlich niedrigere Prävalenzraten haben als die Frauen, weisen die Männer bei der Krankheitskombination Angina pectoris, Diabetes mellitus, Herzinsuffizienz, Hypertonie und Hypercholesterinämie/Hyperlipidämie eine beträchtlich höhere Prävalenzrate auf als Frauen.

- Mehrfacherkrankungen mit Bluthochdruck und Hypercholesterinämie/Hyperlipidämie sind häufiger bei den Männern als bei den Frauen mit einem niedrigeren durchschnittlichen Krankheitsalter verbunden.

Die aufgeführten Merkmale lassen erkennen, daß die gesundheitliche Situation der männlichen Bevölkerung aufgrund niedrigerer Prävalenzraten an multimorbiden Zuständen gegenüber der weiblichen Bevölkerung keinesfalls günstiger zu bewerten ist. Die pauschale Wertung - hohe Krankheitsintensität gleich negativer Gesundheitszustand der Bevölkerung - ist nicht zutreffend [1]. Der Zeitpunkt des Ereignisses "Krankwerden « bzw. die Dauer des Zustandes »Kranksein « lassen sich zwar in der Regel nicht exakt bestimmen, aber ein um mehrere Jahre niedrigeres durchschnittliches Krankheitsalter der männlichen Krankenpopulation deuten auf einen lebenskalendarisch früheren $\mathrm{Be}$ ginn des "Krankwerdens « an polypathischen Zuständen hin. Auch die Dauer des »Krankseins«, beispielsweise als Überlebenszeit nach Diagnosenstellung oder nach Therapiebeginn meßbar, muß bei den mehrfachkranken Männern gegenüber den Frauen kürzer sein: Indikatoren dafür sind die höheren männlichen Prävalenzraten im jüngeren $\mathrm{Er}$ wachsenenalter und die einsetzende $\mathrm{Ab}$ nahme der Prävalenzraten bei den Männern im hohen Alter von 70 bis unter 80 Jahren. Das Vorhandensein der "Multiplizitäten « von Krankheiten, Leiden, Gebrechen und pathologischen Organveränderungen ist bei Männern im höheren Lebensalter immer weniger mit der Überlebensfähigkeit zu vereinbaren. Frauen mit chronischen Krankheiten haben auch höhere Überlebenswahrscheinlichkeiten als Männer [15-17]. Pathogenetisch und symptomatisch gleichartige Krankheiten haben demnach geschlechtsdifferente Verläufe. Prognostisch ungünstigere multimorbide Profile, wie das Zusammentreffen von Hypertonie, Diabetes mellitus, Herzinsuffizienz, Angina pectoris und Hyperlipidämie, kommen in der männ- lichen Krankenpopulation häufiger vor. Wirken mehrere Risikofaktoren gleichzeitig, dann kommt es zu einer Potenzierung der Risiken (Exzeßrisiko), was signifikant zu einer Minderung der ferneren Lebenserwartungswerte bzw. zu einer Erhöhung der Sterblichkeit führen kann. So ist die Übersterblichkeit der Hochdruckkranken besonders hoch, wenn die Krankheit bereits vor dem 50 . Lebensjahr auftritt. Sie wird mit zunehmendem Lebensalter geringer [18, 19]. Dementsprechend wird bei den mehrfachkranken männlichen Hypertonikern ein niedrigeres Krankheitsalter gefunden als bei den weiblichen Hypertonikern.

Die Beziehung zwischen Erkrankungszeitpunkt und Tod infolge von Krankheit hat sich insbesondere in der Neuzeit stark verändert. Im Zuge der epidemiologischen Transition kam es zu einer Ausdehnung des Krankheitsbegriffes und zur Differenzierung pathischer Zustände. Ein verlängertes Überleben von relativ kranken Menschen ist hierbei charakteristisch. Beherrschbare Erkrankungen brauchen keine wesentlichen Funktionseinschränkungen hervorzurufen. Längere Überlebenszeiten der weiblichen Bevölkerung bei chronischen nicht tödlichen Leiden bzw. nach akuten Exazerbationen chronischer Krankheiten, die mit einer Abnahme der Letalität/Fatalität verbunden sind, bewirken auch höhere Prävalenzraten an multimorbiden Zuständen.

Die weibliche Bevölkerung neigt zu einer differenzierteren Wahrnehmung eigener Körperfunktionen und zu einer verstärkten Hinwendung zur eigenen Körperlichkeit und Befindlichkeit [1, 20-24]. Eine niedrigere Schwelle der Symptomtoleranz, ein anderes Gefüge der Definition von »Gesundsein« und "Kranksein« und Tendenzen der Aggravation gesundheitlicher Störungen führen bei Frauen letztendlich zu einem Mehr an Krankheiten.

Zu den Kriterien der Verbesserung von Gesundheitsverhältnissen gehört die Verlängerung der Kankheitsdauer, die Zunahme chronischer Krankheiten sowie die Zunahme der Vielfalt vorkommender Krankheiten. Eine positiv geleitete Explikation der höheren Morbiditätsraten und vermehrt auftretende Funktionseinschränkungen bei der weiblichen Bevölkerung eröffnen einen konsistenten und logischen Zugang zur Erklärung der gesundheitsbezogenen geschlechtsdifferenten Kenngrößen wie Lebenserwartung und Sterblichkeit. Es ist erstaunlich, daß dieser Zusammenhang der universitären "Geschlechterforschung in Deutschland bisher entgangen ist. Frauen vermögen ein höheres Maß an gesellschaftlich-kulturellen Kräften zu mobilisieren, soziale Stützsysteme besser zu nutzen und vorteilhaftere individualpsychologische $\mathrm{Be}$ wältigungsstrategien zu entwickeln, um mit defizitären Situationen des Organismus besser fertig zu werden. Das Gesundheitsverhalten der Geschlechter, das in der Ätiologie vieler Krankheiten eine wichtige Rolle spielt, ist Teil des Sozialverhaltens und als Norm, Tradition und »vererbte« soziale Erfahrung Bestandteil der Kultur. Es läßt sich in der Regel nur im Rahmen längerfristig ablaufender Prozesse des sozialen Wandels beeinflussen. Forderungen nach einer Umverteilung der ohnehin begrenzten Ressourcen zugunsten gesundheitlicher Förderprogramme für die männliche Bevölkerung erscheinen vor diesem Hintergrund weder sinnvoll noch durchführbar. Gesundheitsförderung vermag weder die Verschiedenheit der Geschlechter noch ihrer Lebensprobleme unter einem einheitlichen $\mathrm{Ge}$ sundheitsbild zu harmonisieren. Eine Fokussierung sämtlicher Zielbestimmungen und Handlungskonzepte gesundheitlicher Förderung auf ein ausgesuchtes demographisches Merkmal wie der Geschlechtszugehörigkeit wäre auch ohne Zweifel eine völlig verfehlte Strategie der Gesundheitsvorsorge. Der Widerspruch gesundheiticher Zielbestimmungen und sozialer Attitüden ist ein gesundheitspolitisches Kernproblem, das durch Gesundheitsförderung nicht gelöst werden kann. Ein wünschenswerter Effekt wäre eine Wiederbelebung der gesundheitspolitischen Diskussion über Defizite und notwendige Weiterentwicklung der gesundheitlichen und medizinischen Versorgung der männlichen Bevölkerung. Ziel aller Bemühungen muß es schließlich sein, die gesundheitliche Versorgung zu optimieren und bei begrenzten Ressourcen einen möglichst hohen gesundheitlichen Nutzen für die gesamte Bevölkerung zu erreichen.

\section{Literatur:}

[1] Wiesner, G., Todzy-Wolff, I., und Grimm, J Krankheit und soziale Schicht. In: Ahrens, W., Bellach, B.-M., und Jöckel, K.-H. (Hrsg.): Messung soziodemographischer Mcrkmalc in der Epidemiologie. RKI-Schriften 1/1998. München: MMV Medizin Verlag 1998, $113-140$.

[2] Block, G., Rosenberger, W. F., and Patterson, B. H.: Calorics, fat and cholesterol: Intake patterns in the U.S. populations by race, sex 
and age. Am. J. Pub. Health 78 (1988) 1150-1155.

[3] Enstrom, J. E., Kanim, L. E., and Klein, M. A.: Vitamin $C$ intake and mortality among a sample of the United States population. Epidemiology 3 (1992) 194-202.

[4] Herrmann-Kunz, E., und Thamm, M.: Energie- und Nährstoffaufnahme in den neuen Bundesländern. In: Bellach, B.-M. (Hrsg). Die Gesundheit der Deutschen. Bd. 2. RKIHeft 15/1996, 89-100.

[5] Statistisches Jahrbuch 1995 für die Bundesrepublik Deutschland. Statistisches Bundesamt. Stuttgarr: Metzler-Poeschel Verlag 1995.

[6] Wiesner, G.: Ausgewählte Ergebnisse zur ambulanten Inanspruchnahme und ambulanten Morbidität. Z. ärztl. Fortbild. 79 (1985) 23-24.

[7] Bergmann, E.: Ambulante Inanspruchnahme I. In: Hoffmeister, H., und Bellach, B.-M.: Die Gesundheit der Deutschen. RKI-Heft 7/1995, 174-181.

[8] Bundesministerium für Gesundheit: Statistisches Taschenbuch Gesundheit. Baden-Baden: Nomos-Verlag 1996.

[9] Wiesner, G., Todzy-Wolff, I., und Hoffmeister, H.: Herzinfarkt. In: Hoffmeister, $H$. und Bellach, B.-M: Die Gesundheit der Deutschen. RKI-Heft 7/1995, 1 l-25.
[10] Busfield, J.: Men, women and madness. London: Macmillan press 1996.

[11] Wiesner, G.: Osteoporose - ein prioritäres Gesundheitsproblem mir vielen offenen Fragen. Bundesgesundhbl. 41, 6 (1998) 244-449.

[12] MacQueen, J. B.: Some Methods for Classification and Analysis of Multivariate Observations Proceedings of the Fifth Berkeley Symposium on Mathematical Statistics und Probability 1 (1967) 281-297.

[13] Harrigan, J. A.: Clustering Algorithms. New York: John Whey \& Sons 1975.

[14] Grimm, J., Kant, H., und Wiesner, G.: Multimorbidität. Forschungsbericht. Projekt Nr.: 19. Robert Koch-Institut, Berlin 1997.

[15] Waldron, I.: Sex differences in illness incidence, prognosis and mortality: issues and evidence. Soc. Sci. Med. 17 (1983) 1107-1123.

[16] Lang, E., Arnold, K., und Kupfer, P.: Frauen werden älter - biologische, medizinische und soziologische Ursachen. Z. Gerontol. 27 (1994) $10-15$.

[17] Casper, W., Wiesner, G., und Bergmann, K.: Mortalität in Deutschland - unter besonderer Berücksichtigung der Entwicklung in den alten und neuen Bundesländern. RKI-Heft 10/1995.

[18] Kühne, K., und Brahms, O.: Die Prognose der essentiellen Hypertonie. Darmstadt: Steinkopf 1964.
[19] Jahnecke, J.: Risikofaktor Hypertonie. Hochdruckfibel für die ärztliche Praxis. Studienreihe Boehringer Mannheim. Mannheim: Mannheimer Morgen Großdruckerei und Verlag GmbH 1974.

[20] Schwarzer, R.: Psychologie des Gesundheirsverhaltens. Göttingen: Hogrefe-Verlag 1996.

[21] Barnett, R. C., and Marshall, N. L.: Men, family-role, job-role quality and physical health. Health Psychology 122 (1993) 48-55.

[22] Hibbard, J. H., and Pope, C. R.: The quality of social rcles as predictors of morbidity and mortality. Soc. Sci. Med. 36 (1993) 217-222.

[23] Verbrugge, L. M.: The twain meet: Empirical explanations of sex differences in health and mortality. J. Health Soc. Behv. 30 (1989) 282-304.

[24] Klotz, Th., Hurrelmann, K., und Eickenberg, H.-U.: Der frühe Tod des starken Geschlechts. Deutsches Ärzteblatt 95 (1998) $362-366$.

Verfasser:

Dr. Jürgen Grimm, Edelgard Bittner und Doz. Dr. Dr. Gerd Wiesner, Robert Koch-Institut, Berlin

Korrespondenzanschrift:

Doz. Dr. Dr. G. Wiesner, Robert Koch-Institut, Postfach 650280, 13302 Berlin

\title{
Zum geschlechtsdifferenten Prozeß der Lebensverlängerung in Deutschland
}

\author{
Von G. Wiesner, E. Bittner und I. Todzy-Wolff
}

\section{Zusammenfassung}

Der Lebensverlängerungsprozeß hat sich in diesem Jahrhundert in Deutschland fortgesetzt, wobei die weibliche Bevölkerung einen höheren Zugewinn an Lebenserwartung gegenüber der männlichen Bevölkerung realisieren konnte. Für die Zeitintervalle 1949/51 bis $1960 / 62,1960 / 62$ bis $1970 / 72$, $1970 / 72$ bis $1981 / 83$ und $1981 / 83$ bis 1993/95 wurden für die männliche und weibliche Bevölkerung der Bundesrepublik Deutschland (früheres Bundesgebiet) die relativen Veränderungen der altersspezifischen Überlebenswahrscheinlichkeiten errechnet. Während der Lebensverlängerungsprozeß der weiblichen Bevölkerung über alle Zeitintervalle nahezu kontinuierlich voranschreitet, waren bei der männlichen Bevölkerung in definierten Altersbereichen erhebliche Schwankungen zu beobachten. Der Lebensverlängerungs- prozeß wird bei beiden Geschlechtern zunehmend im höheren Lebensalter wirksam. $\mathrm{Da}$ in den aufeinanderfolgenden Geburtsjahrkohorten die genetisch/biologische Variabilität weitgehend konstant reproduziert wird, ist die geschlechtsdifferente Entwicklung der Lebenserwartung sozialer Natur. Der höhere säkulare Lebenserwartungszugewinn der weiblichen Bevölkerung ist Ausdruck einer höheren Partizipation an der sozialen Aufwärtsentwicklung.

\section{Einführung}

Die Sterbetafeln der männlichen und weiblichen Bevölkerung der Bundesrepublik Deutschland weisen für den Zeitraum der Jahre 1993/95 eine mittlere Lebenserwartung der Männer von 72,99 Jahren gegenüber 79,49 bei den Frauen aus. Männliche Lebendgeborene sterben also, den Sterblichkeitsverhältnissen des Zeitraumes 1993/95 entsprechend, durchschnittlich 6,5 Jahre früher als die gleichzeitig lebendgeborenen Mädchen.

Daß die Sterblichkeit und die Lebenserwartung Unterschiede aufweist, ist keine neue Erkenntnis. Die Lebenserwartung der Frauen war nach den offiziellen Sterbetafeln in den letzten 125 Jahren in Deutschland in allen Altersstufen höher als die der Männer. Die männliche Bevölkerung hat demnach ein generell höheres Sterberisiko als die weibliche Bevölkerung. Die Geschlechtsdifferenzen in der Lebenserwartung zeichnen sich vor dem Hintergrund einer stetigen Zunahme der Lebenserwartung bei beiden Geschlechtern ab. Es stellt sich hier die Frage, ob die männliche Bevölkerung eine verminderte Dynamik des Lebensver- 